\title{
Examining Electron-Boson Coupling Using Time-Resolved Spectroscopy
}

\author{
Michael Sentef, ${ }^{1, *}$ Alexander F. Kemper, ${ }^{2}$ Brian Moritz, ${ }^{1,3,4}$ James K. Freericks, ${ }^{5}$ \\ Zhi-Xun Shen, ${ }^{1,6,7}$ and Thomas P. Devereaux ${ }^{1,7}$ \\ ${ }^{1}$ Stanford Institute for Materials and Energy Sciences (SIMES), SLAC National Accelerator Laboratory, \\ Menlo Park, California 94025, USA \\ ${ }^{2}$ Lawrence Berkeley National Lab, 1 Cyclotron Road, Berkeley, California 94720, USA \\ ${ }^{3}$ Department of Physics and Astrophysics, University of North Dakota, Grand Forks, North Dakota 58202, USA \\ ${ }^{4}$ Department of Physics, Northern Illinois University, DeKalb, Illinois 60115, USA \\ ${ }^{5}$ Department of Physics, Georgetown University, Washington, D.C. 20057, USA \\ ${ }^{6}$ Departments of Physics and Applied Physics, Stanford University, Stanford, California 94305, USA \\ ${ }^{7}$ Geballe Laboratory for Advanced Materials, Stanford University, Stanford, California 94305, USA \\ (Received 5 July 2013; revised manuscript received 2 September 2013; published 26 December 2013)
}

Nonequilibrium pump-probe time-domain spectroscopies can become an important tool to disentangle degrees of freedom whose coupling leads to broad structures in the frequency domain. Here, using the time-resolved solution of a model photoexcited electron-phonon system, we show that the relaxational dynamics are directly governed by the equilibrium self-energy so that the phonon frequency sets a window for "slow" versus "fast" recovery. The overall temporal structure of this relaxation spectroscopy allows for a reliable and quantitative extraction of the electron-phonon coupling strength without requiring an effective temperature model or making strong assumptions about the underlying bare electronic band dispersion.

DOI: 10.1103/PhysRevX.3.041033

Subject Areas: Computational Physics, Condensed Matter Physics

\section{INTRODUCTION}

Pump-probe spectroscopies offer the exciting opportunity to perturb and measure electrons and collective modes in solids on their intrinsic ultrafast time scales. Moreover, they provide a tool to gain insight into the behavior of matter pushed out of its thermodynamic equilibrium state, thereby probing microscopic details of complex manybody systems beyond effective thermodynamic variables. The opportunity, however, comes with a challenge: When the equilibrium concepts of entropy and temperature are overcome, we are also in need of developing a language for true nonequilibrium behavior. Moreover, the value of ultrafast spectroscopies must be enhanced by the ability to relate measurements out of equilibrium to microscopically derivable quantities in an effort to understand dynamics and, ultimately, nonequilibrium itself. In this paper, we address these issues by showing a full nonequilibrium solution of a generic electron-phonon-coupled model system and by relating the temporal spectroscopic response to microscopic quantum-many-body details of the system. We utilize this concept as a basis to directly access the intrinsic decay rates of quasiparticles in the time domain without assumptions about bare-band quantities that are needed in the frequency domain.

\footnotetext{
*sentefmi@stanford.edu
}

Published by the American Physical Society under the terms of the Creative Commons Attribution 3.0 License. Further distribution of this work must maintain attribution to the author(s) and the published article's title, journal citation, and DOI.
Angle-resolved photoemission (ARPES) has been widely used to determine the low-energy electronic structure of a variety of materials [1,2]. Many-body interaction effects are manifest via the self-energy $\Sigma$ giving rise to renormalizations of the electron mass and an overall broadening of the spectral function. As an example, it was thought that ARPES data for the cuprates could thereby be studied to extract the boson coupling that is responsible for high-temperature superconductivity [3-20]. These investigations have provided important insight into the underlying bosons and interactions responsible for kinks in the electronic dispersion.

However, extracting the self-energy and a meaningful electron-boson coupling constant from frequencydependent ARPES data can be challenging. In fact, there is currently no reliable way to extract a frequencydependent electron-phonon coupling. In addition, the interpretation of ARPES data in terms of self-energy naturally rests upon the assumption of an underlying bare electronic band, which becomes renormalized by the coupling to bosonic modes. Similarly, the widths of momentum distribution curves (MDCs) need to be supplemented with a bare Fermi velocity in order to determine scattering rates. Estimates of the dimensionless electronboson coupling strength $\lambda$ therefore vary strongly in the literature, from very small effective scattering rates [21] to extremely strong coupling [18].

As an alternative, a time-domain approach using the relaxation of excited electrons back to equilibrium as a direct technique to measure electron-phonon coupling was proposed by Allen [22]. The basic quantities for this 
method are the deviations from the initial equilibrium values of the electron and phonon occupations in momentum space. A semiclassical Boltzmann approximation that averages over momenta and energy to extract a single energy relaxation time scale was used to extract estimates for electron-phonon couplings from pump-probe photoemission data in simple elemental metals [23,24], but it was also applied to analyze pump-probe data for hightemperature superconductors [25-29].

However, in correlated materials, such as transition metal oxides like the cuprates, or in multiband systems with multiple disconnected Fermi-surface pieces, such as the iron pnictides, the momentum and energy dependences must be resolved since they are crucial ingredients for these materials' interesting properties [30,31]. In addition, a full quantum-mechanical formulation is required to treat arbitrarily strong fields and strong energy and momentumdependent scattering, particularly in materials where the balance between various competing phases may be tipped with the application of strong electric fields. Examples include possible light-induced superconductivity in a stripe-ordered cuprate [32], a dramatically enhanced conductivity in an oxide heterostructure [33], coherent phonon oscillations serving as a marker for a photoinduced phase transition in $\mathrm{VO}_{2}$ [34], the theoretical proposal of dynamical band flipping [35], and pump-probe photoemission, providing a novel classification scheme for charge-density wave insulators in the time domain [36].

In this paper, we combine the strength of the timedomain approach with the power of ARPES self-energy microscopics. We present a microscopic quantum nonequilibrium approach with full momentum and energy resolution that can be applied when effective temperature phenomenology and the semiclassical Boltzmann equation fail and that can be used in conjunction with standard many-body and material-specific computational approaches. Using our model system, we show how the time $(t)$, momentum $(\boldsymbol{k})$, and energy $(\omega)$ resolution provided by state-of-the-art tr-ARPES [25-28,37-47] experiments, in conjunction with the dependence of extracted decay times on the initial-equilibrium sample temperature, provides a direct method to measure the dominant phonon energy and electron-phonon coupling. Hence, we show that relaxational spectroscopy in the time domain allows the possibility to extract detailed information about the system in equilibrium and lends itself to a simple interpretation that does not involve any assumptions about the bare electronic-band dispersion and goes beyond the traditional two-temperature model phenomenology.

The paper is organized as follows. In Sect. II, we outline the electron-phonon coupled-model system and the calculation of double-time Green's functions on the Keldysh contour and the time-resolved photoemission response. In Sec. III, we present our results for the photoexcited electronic structure (Sec. III A), the extraction of the quasiparticle self-energy, and the implied electron-phonon coupling from the temporal relaxation of the electrons (Sec. III B). Section IV contains a discussion of the results and their implications. Additional considerations regarding the trade-off between time and energy resolution, the breakdown of an effective temperature description, the connection between relaxation rates and the self-energy, and the additional effect of including electron-electron scattering are provided in Ref. [48].

\section{MODEL AND METHOD}

\section{A. Introductory remarks}

For the formulation of the nonequilibrium problem, we employ the well-established Keldysh Green's function technique. The single-particle properties of the electrons are described by the double-time Green's function $G\left(t, t^{\prime}\right)$ on the complex Keldysh contour. The Green's function encodes the electrons' initial equilibrium state, their real time evolution during and after a perturbation via a pump laser pulse, and their memory of the initial state. The double-time Green's functions allow us to extract the relevant information (occupation of states, tr-ARPES response) about the excited-electron system in a microscopic way.

The pump laser field of arbitrary temporal shape is included as a space-independent and time-dependent vector potential $\boldsymbol{A}(t)$ in a nonperturbative way via Peierls substitution $[49,50]$. This approach was formulated earlier by Jauho and Wilkins [50], and we extend it numerically to longer time scales and arbitrary field strengths. The coupling of the electrons to a nondispersive optical phonon mode is modeled by the well-established and controlled diagrammatic Migdal approximation [51]. The resulting integro-differential equations of motion for the Green's functions [52] are solved using massively parallel numerical integration. The code is parallelized easily since the equations for different momentum points decouple in the discretized Brillouin zone. An additional Fourier transform of the lesser component of the resulting interacting Green's function is performed to extract the tr-ARPES spectra [53]. In this step, a shape function is introduced to take the probe-pulse width into account. Additionally, the momenta need to be time shifted depending on the pump field to ensure gauge invariance [54].

\section{B. Model}

We study the Holstein Hamiltonian [55]

$H=\sum_{\boldsymbol{k}} \epsilon(\boldsymbol{k}) c_{\boldsymbol{k}}^{\dagger} c_{\boldsymbol{k}}+\Omega \sum_{i} b_{i}^{\dagger} b_{i}-g \sum_{i} c_{i}^{\dagger} c_{i}\left(b_{i}+b_{i}^{\dagger}\right)$,

where the individual terms are the electronic kinetic energy with dispersion $\epsilon(\boldsymbol{k})$, the phonon energy for dispersionless Einstein phonons of frequency $\Omega$, and the electron-phonon interaction, respectively. Here, $c_{\boldsymbol{k}(i)}^{(\dagger)}$ is the electronic annihilation (creation) operator in momentum space (real 
space), $b_{i}^{(\dagger)}$ is the bosonic phonon annihilation (creation) operator on lattice site $i$, and $g$ is the momentumindependent electron-phonon coupling strength.

The starting bare nonequilibrium Green's function [54]

$$
G_{\boldsymbol{k}}^{0}\left(t, t^{\prime}\right)=i\left[n_{F}(\boldsymbol{\epsilon}(\boldsymbol{k}))-\theta_{c}\left(t, t^{\prime}\right)\right] \exp \left[-i \int_{t^{\prime}}^{t} d \bar{\epsilon} \boldsymbol{\epsilon}(\boldsymbol{k}-\boldsymbol{A}(\bar{t}))\right],
$$

where $n_{F}(\omega)=1 /(1+\exp (\omega / T))$ is the Fermi function at temperature $T, t$ and $t^{\prime}$ are times on the Keldysh contour $\mathcal{C}$, and $\theta_{c}\left(t, t^{\prime}\right)$ is the Heaviside function on the contour, which equals 1 if $t$ is later on the contour than $t^{\prime}$ and 0 otherwise. $\epsilon(\boldsymbol{k})$ is the single-particle energy dispersion for a square lattice with nearest-neighbor $\left(V_{\mathrm{nn}}\right)$ and next-nearestneighbor $\left(V_{\mathrm{nnn}}\right)$ hopping,

$$
\boldsymbol{\epsilon}(\boldsymbol{k})=-2 V_{\mathrm{nn}}\left(\cos k_{x}+\cos k_{y}\right)+4 V_{\mathrm{nnn}} \cos k_{x} \cos k_{y}-\mu,
$$

measured with respect to the chemical potential $\mu$. Here, we use the convention that $\hbar=c=e=1$, and we work in the Hamiltonian gauge; i.e., the scalar potential is set to zero.

We incorporate the electron-phonon interactions in the Migdal limit, which is appropriate for weak coupling. In this case, the electronic self-energy due to electron-phonon coupling is

$$
\Sigma\left(t, t^{\prime}\right)=i g^{2} \sum_{\boldsymbol{k}} D^{0}\left(t, t^{\prime}\right) G_{\boldsymbol{k}}^{0}\left(t, t^{\prime}\right) .
$$

The bare-phonon Green's function $D^{0}\left(t, t^{\prime}\right)$ is [56]

$$
\begin{aligned}
D^{0}\left(t, t^{\prime}\right)= & -i\left[n_{B}(\Omega)+1-\theta_{c}\left(t, t^{\prime}\right)\right] \exp \left[i \Omega\left(t-t^{\prime}\right)\right] \\
& -i\left[n_{B}(\Omega)+\theta_{c}\left(t, t^{\prime}\right)\right] \exp \left[-i \Omega\left(t-t^{\prime}\right)\right],
\end{aligned}
$$

where $n_{B}(\Omega)=1 /[\exp (\Omega / T)-1]$ is the Bose function.

\section{Method}

With the self-energy above, we solve the Dyson equation for the interacting Green's function $G$,

$G_{\boldsymbol{k}}\left(t, t^{\prime}\right)=G_{\boldsymbol{k}}^{0}\left(t, t^{\prime}\right)+\int_{\mathcal{C}} d t_{1} d t_{2} G_{\boldsymbol{k}}^{0}\left(t, t_{1}\right) \Sigma\left(t_{1}, t_{2}\right) G_{\boldsymbol{k}}\left(t_{2}, t^{\prime}\right)$.

This can be done by casting the Dyson equation as a matrix equation $[57,58]$. As an alternative, the Dyson equation can be rewritten by expanding the integral with Langreth rules, which gives equations of motion for the Matsubara, retarded, real-imaginary, and lesser Green's functions $[52,59]$. This result leads to a set of Volterra integrodifferential equations that can be solved via standard numerical integration. For the results presented here, we have used the Volterra scheme. Its main advantages are that the equations are manifestly causal and that the required memory is reduced by the use of symmetries.
The pulse that is of direct interest to pump-probe experiments is, by nature, a propagating light pulse; this implies an oscillating field without a zero-frequency component. We model the pump via an oscillating vector potential along the (11) or Brillouin-zone diagonal direction with a Gaussian profile,

$$
\boldsymbol{A}(t)=(\hat{\boldsymbol{x}}+\hat{\boldsymbol{y}}) \frac{E_{\mathrm{max}}}{\omega_{p}} \sin \left(\omega_{p} t\right) \exp \left(-\frac{\left(t-t_{0}\right)^{2}}{2 \sigma^{2}}\right) .
$$

The tr-ARPES intensity for a probe pulse of width $\sigma_{\mathrm{pr}}$ is computed from [53]

$$
\begin{aligned}
I\left(\boldsymbol{k}, \omega, t_{0}\right)= & \operatorname{Im} \frac{1}{2 \pi \sigma_{\mathrm{pr}}^{2}} \int d t \int d t^{\prime} \tilde{G}_{\boldsymbol{k}}^{<}\left(t, t^{\prime}\right) e^{-\left(t-t_{0}\right)^{2} / 2 \sigma_{\mathrm{pr}}^{2}} \\
& \times e^{-\left(t^{\prime}-t_{0}\right)^{2} / 2 \sigma_{\mathrm{pr}}^{2}} e^{i \omega\left(t-t^{\prime}\right)}
\end{aligned}
$$

where the integral is along real times, and each component $k_{i}$ of the momentum argument of $\tilde{G}_{\boldsymbol{k}}^{<}\left(t, t^{\prime}\right)$ is shifted $\left[k_{i} \rightarrow\right.$ $k_{i}+\frac{1}{\bar{t}^{\prime}-t} \int_{t}^{t^{\prime}} d \bar{t} A_{i}(\bar{t})$, where $A_{i}$ is the corresponding $i$ th component of the vector potential] to restore gauge invariance [54]. In Ref. [48], we illustrate the importance of the temporal probe-pulse width $\sigma_{\mathrm{pr}}$ for the effective energy resolution of spectral features.

\section{Setup and model parameters}

We consider 2D tight-binding electrons in a partially filled metallic band with parameters $V_{\mathrm{nn}}=0.25 \mathrm{eV}$, $V_{\mathrm{nnn}}=0.075 \mathrm{eV}$, and $\mu=-0.255 \mathrm{eV}$, chosen to approximate a Fermi surface as seen in the cuprates at high dopings where the pseudogap is negligible. The dispersionless Holstein phonons have a frequency $\Omega=0.1 \mathrm{eV}$ and electron-phonon coupling $g=\sqrt{0.02} \mathrm{eV}$ as representative values for optical phonons in the cuprates. In equilibrium, the phonon frequency sets the relevant energy scale at which a kink at $\pm \Omega$ in the band structure occurs [60], as shown in Figs. 1(a)-1(c). The dimensionless coupling constant $\lambda$ is a measure of the strength of lattice distortion relative to the kinetic energy of the electrons, and it is defined via the slope of the real part of the self-energy that determines the electronic mass renormalization. Our choice of parameters corresponds to a value of $\lambda=0.42$, which is sufficiently large to produce a visible kink in the dispersion for our balance of energy and time resolution but still small enough to justify the use of the Migdal approximation for the electron-phonon coupling.

For the electric pump and probe fields, we choose parameters that allow us to compute the full time evolution on the Keldysh contour by the solution of the integrodifferential equations with a reasonable computational effort. Specifically, we choose a Gaussian field envelope of width $\sigma=4$ femtoseconds (fs) and a center frequency of $0.5 \mathrm{eV}$, which is smaller than UV pump pulses around $1.5 \mathrm{eV}$ that are currently typically used in tr-ARPES experiments. The smaller frequency affects the transient 

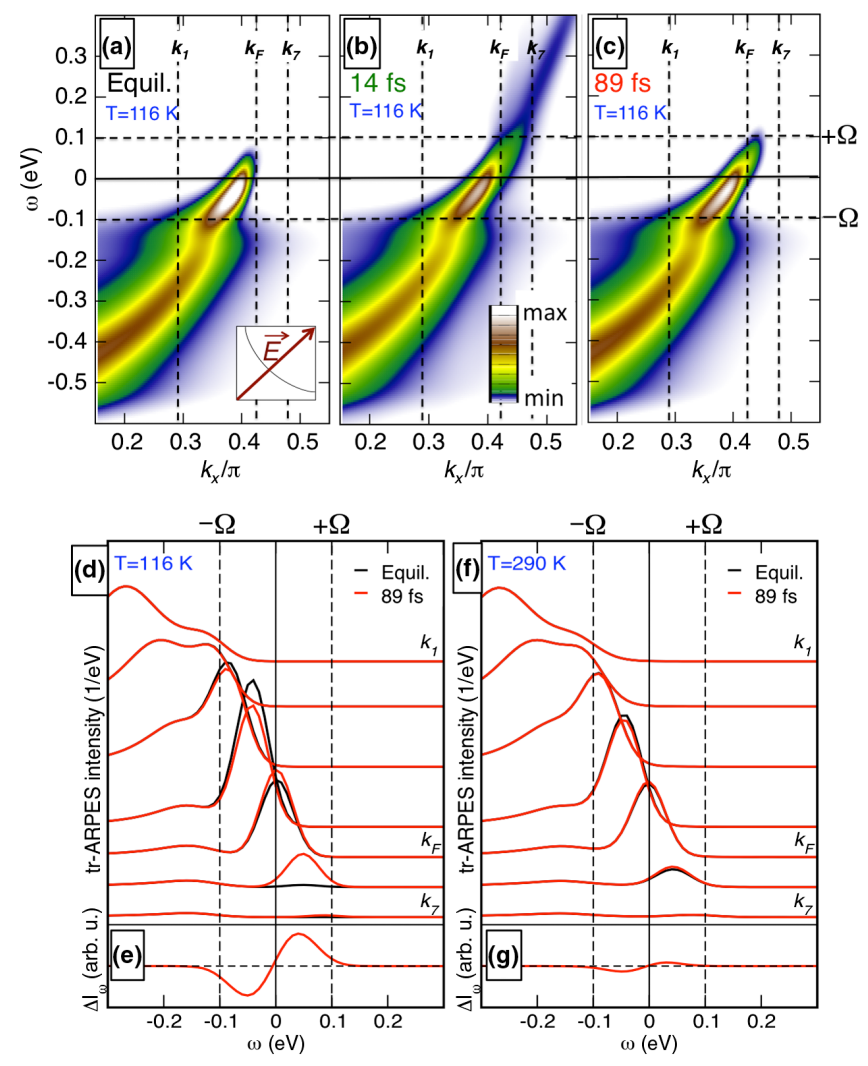

FIG. 1. $\boldsymbol{k}$-resolved photoexcited electronic structure. (a) Equilibrium tr-ARPES intensity at temperature $T=116 \mathrm{~K}$ for a cut along the Brillouin zone diagonal. The pump-field polarization is aligned along this direction (inset). Horizontal dashed lines mark the position of "kinks" in the electronic dispersion at $\pm \Omega$. (b) Map of the tr-ARPES intensity shortly (14 fs) after the pump pulse and (c) at a later delay time $t=$ 89 fs. The system has partially relaxed toward equilibrium outside the energy range $\pm \Omega$, but quasiparticles remain excited within this range. (d) EDCs corresponding to the data shown in (a, equilibrium) and (c, 89 fs) for momenta $\boldsymbol{k}_{1}$ to $\boldsymbol{k}_{7}$ as indicated in panels (a) and (c). For clarity, EDCs are vertically shifted. (e) Intensity difference $\Delta I_{\omega}$ between photoexcited (89 fs) and equilibrium tr-ARPES data. (f-g) EDCs and spectral changes [same as in (d-e)] for a higher initial equilibrium temperature $T=290 \mathrm{~K}$, showing that the time for recovery shortens for increased temperature.

behavior quantitatively. However, it allows us to use a coarser time-step discretization and therefore to observe the relaxation of the excited electrons for a longer time, while it does not affect our conclusions about relaxation effects. The delay time is defined with respect to the center of the Gaussian pulse envelope. The electric field has a peak strength $E_{\max }=0.4 \mathrm{~V} / a_{0}$. While this field strength is at the high end of what can be currently achieved experimentally, this choice is motivated by the fact that it leads to relatively strong, clearly visible spectral redistributions whose temporal decay can be followed easily. Again, the details of the pump do affect the results but not the conclusions. This is explicitly demonstrated in Ref. [48] by analyzing the relaxation times for different maximal pumpfield strengths.

The subsequent tr-ARPES probe pulse has a Gaussian envelope width of $\sigma_{\mathrm{pr}}=16.5 \mathrm{fs}$. This choice is motivated by the maximum contour length that can be reached with reasonable computational effort, since the earliest and latest tr-ARPES observation delay times for which one can extract spectra have to fulfill a $3 \sigma_{\mathrm{pr}}$ minimum distance to the actual earliest and latest times on the contour. In Ref. [48], we also show the equilibrium tr-ARPES spectra for smaller $\sigma_{\mathrm{pr}}$, which enables a better time resolution but necessarily leads to worse energy resolution.

In the simulations, energy scales are measured in units of $\mathrm{eV}$, which implies a natural time scale of $\mathrm{h} / \mathrm{eV} \approx 0.658 \mathrm{fs}$. While the scales are fixed here for clarity, one should keep in mind that a simple rescaling can be applied; e.g., doubling all the time scales while dividing the energy scales in half preserves all the relations.

\section{RESULTS}

\section{A. Photoexcited electronic structure: Energy- and temperature-dependent relaxation}

In Fig. 1, we show the energy- and momentum-resolved tr-ARPES spectra along a nodal cut for three delay times before [Fig. 1(a)], during [Fig. 1(b)], and after the pump pulse [Fig. 1(c)]. The pump pulse drives electrons from below the Fermi level $E_{F}$ to empty states above $E_{F}$ [Fig. 1(b)] as the system absorbs energy leaving the electrons in a photoexcited state. The excess energy of the electrons is then transferred into the bath of phonons, and the electrons relax back towards equilibrium [Fig. 1(c)]. Figure 1(d) provides a comparison of the $\boldsymbol{k}$-resolved energy-distribution curves (EDC) before and 89 fs after the pump. Clearly, the phonon window $\mathcal{W}=$ $[-\Omega, \Omega]$ around the Fermi level sets an energy scale for persistent spectral changes: While electronic quasiparticles outside this window have already relaxed back to their equilibrium EDC, the quasiparticles inside remain excited at the particular time delay shown here. This phonon-window effect is highlighted in the momentum-integrated pumpinduced spectral changes in Fig. 1(e): Photoexcited electrons above $E_{F}$ and holes below $E_{F}$ only persist inside the window set by the phonon frequency $\Omega$.

In equilibrium at zero temperature, kinematics prevents electron relaxation unless the quasiparticles have sufficient energy to emit phonons, which is why this window is expected. In Fig. 1(f), we show that raising the initial equilibrium temperature leads to smaller spectral changes compared to Fig. 1(d) at the same time delay. Correspondingly, the $\boldsymbol{k}$-integrated spectral changes shown in Fig. 1(g) are significantly smaller than the ones in Fig. 1(e) (shown on the same vertical scale), although the phonon-window effect is still visible.

We note here that this result is quite reminiscent of electronic relaxation measured in graphene [61-65] as 
well as in an optimally doped cuprate superconductor in the normal and superconducting states $[27,66]$. The phonon-window effect provides guidance towards a quantitative way of understanding binding-energy-resolved pump-probe decay rates in terms of the equilibrium selfenergy. Before presenting the details of this analysis, we first show that a more traditional way of interpreting pumpprobe spectroscopies, the "hot-electron" model, is neither necessarily appropriate for describing the nonequilibrium transient state nor a prerequisite for our method to work. The hot-electron model assumes that the laser pump initially raises the temperature of the electrons in a hot quasithermal state practically decoupled from the lattice. The quasithermalization of electrons is facilitated on short time scales by electron-electron scattering, leading to distinct electronic and lattice "temperatures" in twotemperature models $[22,67]$. The electrons then transfer their excess energy to the lattice until the electronic and lattice subsystems equilibrate.

For our model system, the hot-electron picture does not provide a valid description of the photoexcited electrons, which is demonstrated in Video 1, because we do not have any electron-electron scattering to allow for the fast relaxation of the electrons into a thermal distribution. In parts (a) and (b) of Video 1, we demonstrate the influence of the electric field strength by showing the momentumresolved tr-ARPES spectrum along the diagonal momentum cut at a short time delay (1 fs) for two different pump pulses. Part (a) of Video 1 is for the same maximum field strength $E_{\max }=0.4 \mathrm{~V} / a_{0}$ that was used in Fig. 1; part (b) of Video 1 is for a 10-times-stronger field. The weaker pump mainly excites electrons within the bare energy band, but the stronger pump clearly disrupts the band structure and induces ladderlike structures that are reminiscent of Wannier-Stark ladders in strong dc electric fields $[68,69]$.

The breakdown of the hot-electron picture becomes evident when we integrate the tr-ARPES spectra in the momentum window around the Fermi momentum between $\boldsymbol{k}_{1}$ and $\boldsymbol{k}_{7}$ (see Fig. 1). The energy-distribution curves in Fig. 2 are not simply featureless decaying exponentials, as they would be if the hot-electron model was valid, but they show distinct higher-energy peaks related to the pump characteristics. Although it is possible to extract an effective temperature from the linear slope of the logarithmic intensities in a very small energy window $(<0.1 \mathrm{eV})$ near the Fermi level (see Fig. S3 in Ref. [48]), this "temperature" does not provide a meaningful description of the photoexcited system. In particular, it fails to account for the actual excess energy in the electronic subsystem due to the spectral features at higher energies. In fact, once this high spectral weight has dissipated, a fit to an effective Fermi distribution shows that the increase in the effective temperature is very small, much smaller than that obtained from forcing a fit at shorter time delays (see Ref. [48]).
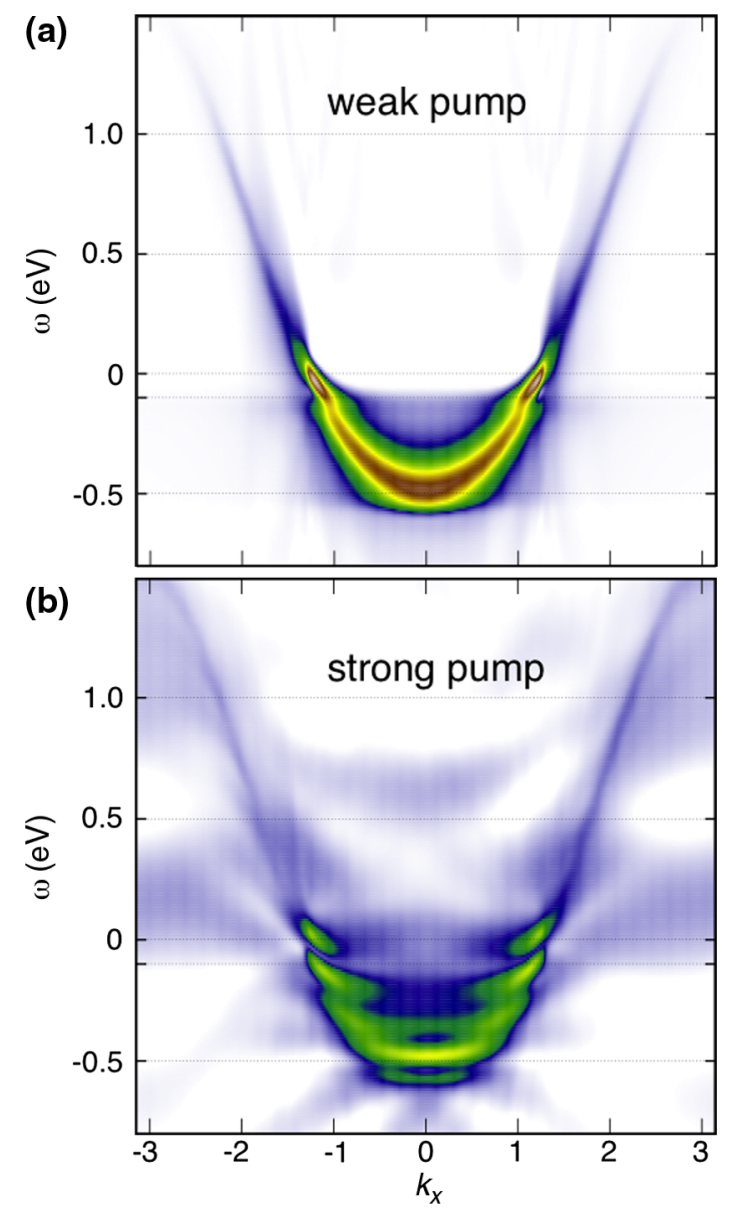

VIDEO 1. Video demonstration of pumping electrons with different driving field strengths. Note that the time units in the movies are 1 time unit $=0.658$ fs. The figures show snapshots at a pump-probe delay time of 1 fs: (a) tr-ARPES intensity map (delay time of $1 \mathrm{fs}$ ) for a weak pump field (same as in Fig. 1) with maximum field strength $E_{\max }=0.4 \mathrm{~V} / a_{0}$. (b) A 10-timesstronger pump field $\left(E_{\max }=4 \mathrm{~V} / a_{0}\right)$. For a lattice constant $a_{0}=3.4 \AA$, these peak field strengths are $\approx 12 \mathrm{MV} / \mathrm{cm}$ ("weak pump") and $118 \mathrm{MV} / \mathrm{cm}$ ("strong pump"), respectively.

These nonthermal features are even more pronounced for the strong pump. This observation clearly shows that photoexcitation is not necessarily the same as heating and that a full nonequilibrium modeling beyond effectivetemperature phenomenology is often essential for correctly describing pump-probe spectroscopies. Since we include only electron-phonon scattering in our simulation and neglect electron-electron scattering, the discrepancy between quasithermal behavior and the observed transient spectra is not too surprising. The inclusion of electronelectron scattering leads to a closer resemblance of spectra with two-temperature phenomenology (see additional calculations, including electron-electron scattering, in Ref. [48]), but this is not guaranteed to be the case. Moreover, even if it is the case, the single relaxation rate 


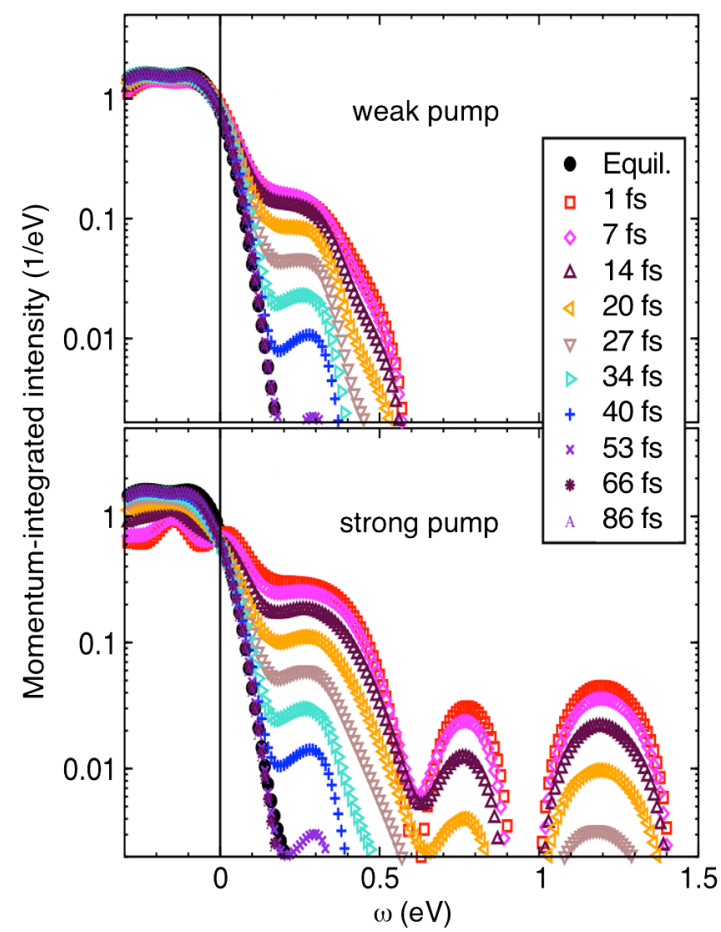

FIG. 2. Weak versus strong pump field. Energy distribution curves integrated over momenta along the Brillouin zone diagonal (same momentum window $\boldsymbol{k}_{1}$ to $\boldsymbol{k}_{7}$ close to $\boldsymbol{k}_{F}$ as indicated in Fig. 1) for various pump-probe delay times.

from two-temperature fits is not simply connected to energy-dependent population decay rates, as we show in Ref. [48]. Therefore, since tr-ARPES experiments probe the full energy- and momentum-resolved population

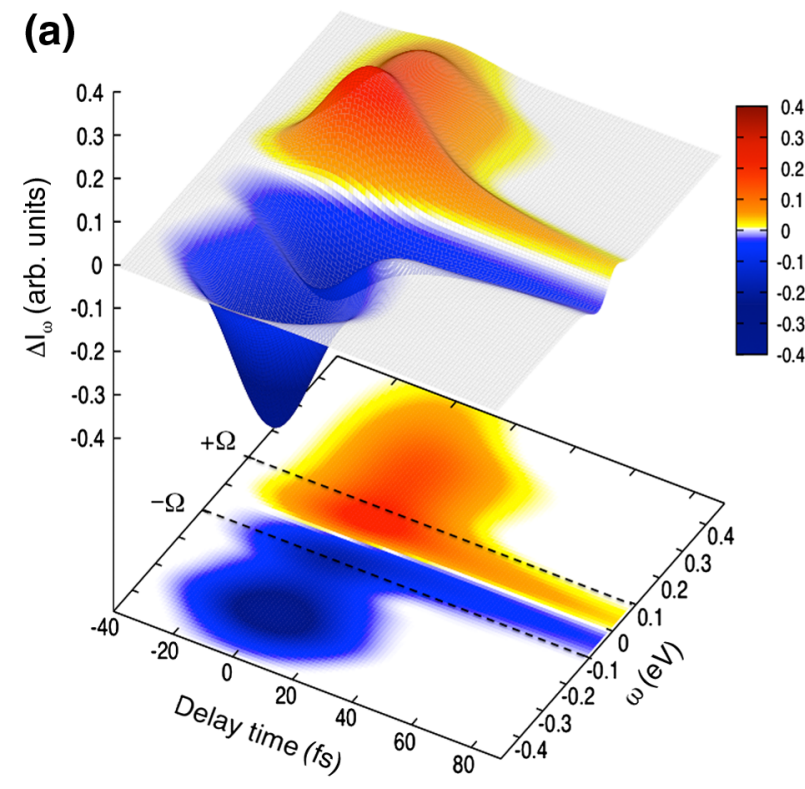

dynamics, the data analysis should go beyond effectivetemperature decay rates.

\section{B. Extracting equilibrium system properties by pump-probe photoemission}

We now focus on a quantitative analysis of energydependent relaxation rates for the weaker pump and the higher initial temperature $T=290 \mathrm{~K}$. Figure 3(a) shows the pump-induced spectral changes relative to the initial state, integrated along momenta on the Brillouin zone diagonal, in a continuous map as a function of energy and delay time. Here, the phonon-window effect becomes even more obvious: Persistent excitations of electrons and holes are only observed inside $\mathcal{W}$, whereas photoexcited electrons outside $\mathcal{W}$ decay on a much faster time scale. The dashed lines mark the phonon window $\mathcal{W}$, which clearly sets the relevant energy scale for slow versus fast relaxation. In Fig. 3(b), we present cuts for selected energies of the same data as in Fig. 3(a): Both electrons and holes inside $\mathcal{W}$ decay on a significantly longer time scale than their counterparts outside $\mathcal{W}$. The slight asymmetry between holes and electrons (negative and positive binding energies) is related to asymmetry in the underlying band structure, which is made clear in the discussion that follows.

We fit decaying exponentials [solid lines in Fig. 3(b)] to these curves, starting at a delay time of $40 \mathrm{fs}$, chosen to be sufficiently large such that pump and probe pulses do not significantly overlap. From these fits, we extract the trARPES relaxation rates shown in Fig. 4(a) on a logarithmic scale. The relaxation rates show a steep decline inside the

\section{(b)}

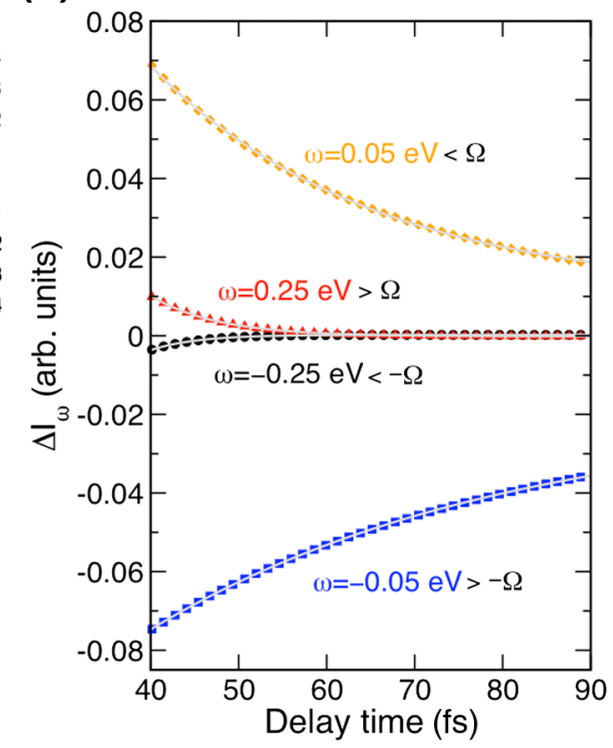

FIG. 3. Transient relaxation of the pump-induced spectral intensity changes for equilibrium temperature $T=290 \mathrm{~K}$. (a) Spectral changes as a function of pump-probe delay time integrated over momenta along the whole Brillouin zone diagonal. (b) Same data as in (a) for selected energies inside $(0.05 \mathrm{eV})$ and outside $(0.25 \mathrm{eV})$ the phonon window for positive (electrons) and negative (holes) binding energies. 
(a)

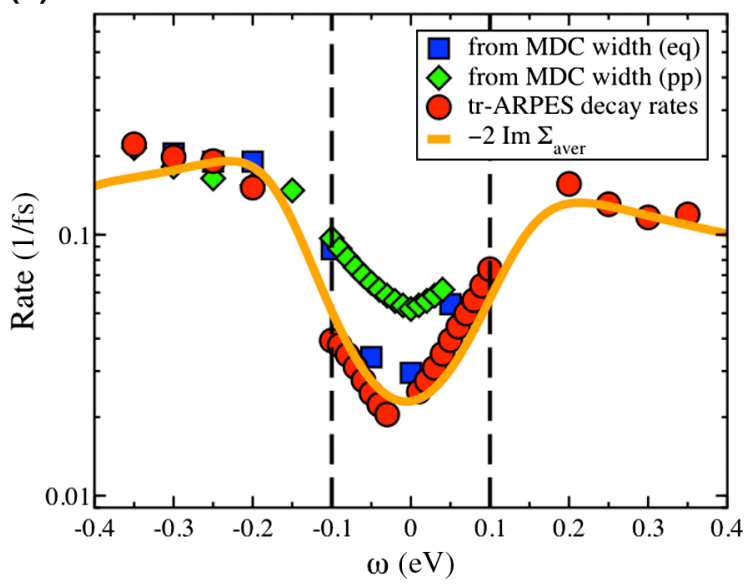

(b)

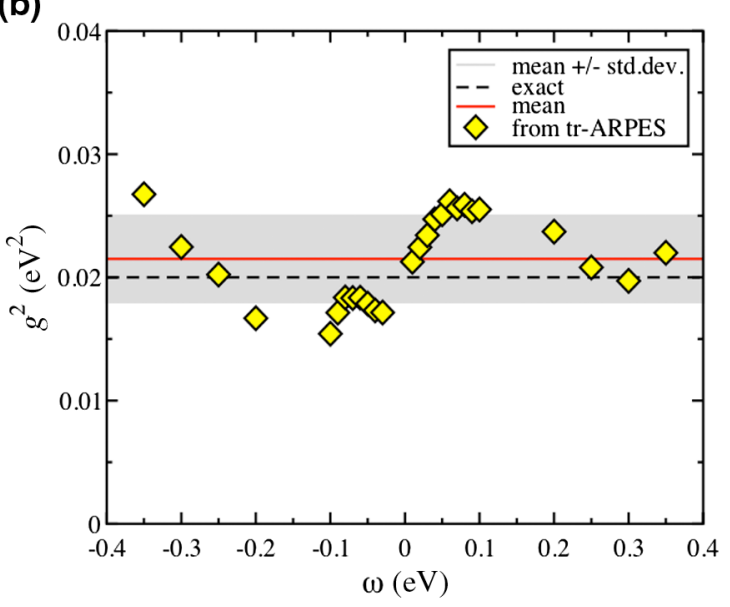

FIG. 4. Energy-dependent relaxation due to electron-phonon scattering. (a) Relaxation rates from tr-ARPES (red circles) compared with equilibrium MDC widths for a continuous probe beam as in an equilibrium measurement ("eq," blue squares) and pump-probe setup ("pp," $\sigma_{\mathrm{pr}}=16.5 \mathrm{fs}$ probe pulse, green diamonds). The orange line shows the corresponding equilibrium electron-phonon scattering rate, taking into account energy resolution (see Ref. [48]). (b) Extraction of the electron-phonon coupling strength from the ratio of tr-ARPES decay rates and equilibrium self-energy (see Ref. [48]). The red solid line shows the mean value of the data points. The black dashed line indicates the exact input value of $g^{2}$. The grey shaded area shows the confidence interval estimated from the statistical standard deviation of the data.

phonon window. This characteristic behavior is indeed reminiscent of the equilibrium scattering rates (selfenergy) due to coupling between electrons and an optical phonon branch, reflected in a suppression of the imaginary part of the equilibrium self-energy in the phonon window. To make this connection between tr-ARPES relaxation rates and the equilibrium self-energy apparent, we plot $-2 \operatorname{Im} \Sigma_{\text {aver }}$ in Fig. 4(a). For comparison, we account for energy resolution due to the probe-pulse width by an additional averaging of relaxation times appropriate for the pump-probe setup (see Ref. [48]).

The good agreement between the tr-ARPES relaxation rates and the equilibrium self-energy is the central result of our paper. It indicates that the return to equilibrium of an electron excited into a state of energy $\omega$ and momentum $\boldsymbol{k}$ is solely determined by the equilibrium quasiparticle selfenergy $\Sigma(\boldsymbol{k}, \omega)$. This result suggests that relaxational pump-probe photoemission spectroscopy can be used as an effective tool for accurately measuring equilibrium system properties in a direct way in the time domain without resorting to any assumptions about the underlying bare-band structure. Traditionally, equilibrium ARPES experiments are used to extract an estimate for electronic lifetimes through the linewidths of momentum distribution curves (MDCs). For optimal energy and momentum resolution and only a single source of decoherence, the full width at half maximum (FWHM) is related to the relaxation rate at the corresponding energy via $\tau^{-1}(\omega)=$ $\operatorname{FWHM}(\omega) v_{F}$, where $v_{F}$ is the bare Fermi velocity along the corresponding momentum cut and the equation is valid near the Fermi level for a linear band dispersion. In order to compare relaxation rates extracted from MDC widths directly for the same energy resolution as the tr-ARPES decay rates, we extract the MDC widths from a tr-ARPES snapshot using the same probe width $\left(\sigma_{\mathrm{pr}}=16.5 \mathrm{fs}\right)$ as for the pump-probe simulation, as well as for a continuous probe beam as in a true equilibrium ARPES experiment. The continuous probe equilibrium MDC widths show good agreement with the tr-ARPES decay rates, but the MDC linewidths for the pump-probe setup with restricted energy resolution imply larger rates inside $\mathcal{W}$ [see Fig. 4(a)]. This can be understood from the fact that when the relaxation rates and, therefore, the MDC widths vary strongly with binding energy, the energy resolution has a crucial effect on the effective imaginary part of the self-energy extracted from MDC widths.

It might seem counterintuitive that a system out of equilibrium relaxes at the equilibrium scattering time scale, determined by the imaginary part of the self-energy, but this result should hold in nearly all systems at long times, provided that the system relaxes back to an equilibrium state. This result has been shown for single-time kinetic equations using a relaxation time ansatz [22,70] (see also the Appendix of Ref. [71]). However, by construction, a single-time formalism is incapable of addressing the momentum- and energy-dependent relaxation rates measured with pump-probe photoemission spectroscopy. As we have shown, the energy dependence is crucial to extract information about dominant bosonic mode couplings that appear as the phonon-window effect. Both the computation of the tr-ARPES response and the connection of its relaxation rates with the dynamical equilibrium self-energy require the full double-time Green's function formalism used in this work. 
For the full double-time Green's functions, one can show that nonequilibrium relaxation is governed by the equilibrium self-energy along the following lines: For small deviations from equilibrium, the change of the spectrum is second order in the deviation from equilibrium, while the change of the occupation is first order, primarily because of the change of the distribution function from the equilibrium Fermi-Dirac distribution [70,72]. This result arises from a linearization of the quantum Boltzmann equation for small perturbations from equilibrium and explains why linear response coefficients can be calculated from equilibrium response functions. Hence, the Green's functions should be the same as in equilibrium, except for a small change of the distribution function. If the self-energy of the system in equilibrium does not depend too strongly on temperature, then it is weakly dependent on the distribution function, and as a result, we will see the nonequilibrium system relax back to equilibrium at the longer time scales via the equilibrium relaxation rates. These conditions are usually fulfilled for sufficiently high temperatures. Care has to be taken when the temperature becomes so low compared to the lowest relevant bosonic mode energy that relaxation via the equilibrium self-energy is completely blocked at low binding energies. In this case, relaxation pathways of higher order in the electron-boson coupling become relevant for the long-time relaxation, corresponding to effects of higher order in the deviation from the equilibrium distribution.

We now show how more detailed knowledge about the electron-phonon scattering processes allows us to quantitatively extract the electron-phonon mode coupling strength from a comparison of the tr-ARPES relaxation rates $\Gamma_{\text {tr }}(\omega)$ and the self-energy $\Sigma(\omega)$. To this end, we treat our tr-ARPES relaxation rates as "experimental data" and assume that we know the line shape of $\operatorname{Im} \Sigma(\omega)$. Within the Migdal approximation to the Holstein model, the relaxation rate is proportional to $g^{2}$ and of the form $\Gamma(\omega)=g^{2} \phi(\omega)$ (see Ref. [48]). The function $\phi(\omega)$ reflects electron-phonon scattering processes and takes into account the available scattering phase space related to the electronic-band structure via the local electronic density of states $N(\omega)$. This is the reason why an asymmetry in the band structure results in an electron-hole asymmetry in the relaxation rates.

The measured tr-ARPES relaxation rates $\Gamma_{\text {tr }}$ are naturally convolved with the probe-pulse shape and therefore include an additional energy averaging, since there is a trade-off between time resolution and energy resolution. Therefore, we apply an energy-averaging procedure to $\phi(\omega)$ (see Ref. [48]), which yields $\phi_{\text {aver }}$. The electronphonon coupling strength is then extracted from $g^{2}=$ $\Gamma_{\text {tr }}(\omega) / \phi_{\text {aver }}(\omega)$. Figure 4(b) shows this ratio as a function of energy, which serves as an a posteriori estimate of $g^{2}$ given the numerical simulation data. The extracted mean value, including an error estimate from the statistical stan- dard deviation, is $g^{2}=0.021 \pm 0.004 \mathrm{eV}^{2}$, to be compared with the exact input value $g^{2}=0.02 \mathrm{eV}^{2}$. This agreement highlights the fact that a nonequilibrium measurement can serve as an accurate tool for extracting equilibrium system properties, a feat that may be difficult to accomplish by pure equilibrium measurements.

\section{DISCUSSION}

The central result of this paper is that equilibrium ideas can be used to understand the nonequilibrium dynamics of a pumped system. This is facilitated by the connection between relaxation rates for the decay of photoexcited electrons and the equilibrium electron-phonon scattering rates. Importantly, both the dependence of the rates on the binding energy and the dependence on the initial equilibrium temperature affect the result but not the conclusion. We have shown that, similar to the situation for driven strongly correlated electrons [73], a simplified modeling in terms of effective temperatures does not provide an adequate description of photoexcited systems with full nonequilibrium quantum dynamics. This may partly be due to the neglect of electron-electron scattering in our study. However, even if the effective-temperature-model fits work, they reduce population dynamics into a single decay rate. Here, we have presented a more appropriate way of analyzing the full wealth of momentum- and energydependent population dynamics measured by time- and angle-resolved photoemission spectroscopy.

The relation between the equilibrium self-energy and the nonequilibrium relaxation makes pump-probe spectroscopies a convenient tool to disentangle equilibrium decoherence pathways. As a consequence, the strength of electron-phonon couplings can be extracted from pump-probe relaxation times with a high level of accuracy. The presence of electron-electron interactions is expected to lead to richer short-time transient behavior, but it supports this conclusion, provided that the electronic system relaxes back to an equilibrium state at long times by dissipating the pump energy into the lattice. This behavior is shown in Ref. [48] by adding electron-electron interactions as an additional scattering channel. For dispersive boson modes like acoustic phonons, the window effect is not as pronounced as for the Holstein mode discussed in this paper, but the same simulation technique and analysis can be applied. The same is true for multiple bosonic modes coupled to the electrons, each of which will show up as a characteristic change of the decay rate around the respective mode energy. Finally, our approach can also be applied to general electron-boson interactions, for instance, coupling to magnons. Therefore, this approach could potentially be used to better characterize the bosonic couplings in high-temperature superconductors.

As a final remark, the recent work by Kemper et al. [71] provides a complementary point of view by demonstrating that time-domain Compton scattering measures 
characteristic oscillations of the time-dependent momentum distribution. These oscillations correspond to energy shifts due to bosonic mode couplings and thereby provide information on the real part of the self-energy, which is related through analyticity by the Kramers-Kronig relations to the imaginary part. Since the imaginary part is measured by time-resolved photoemission as discussed in the present work, the two works together show the power of time-resolved spectroscopies, which demonstrably bear the potential to pinpoint the full nontrivial many-body dynamics of complex quantum systems beyond equilibrium probes.

\section{ACKNOWLEDGMENTS}

This work was supported by the Department of Energy, Office of Basic Energy Sciences, Division of Materials Sciences and Engineering (DMSE) under Contracts No. DE-AC02-76SF00515 (Stanford/SIMES), No. DEFG02-08ER46542 (Georgetown), and No. DE-SC0007091 (for the collaboration). Computational resources were provided by the National Energy Research Scientific Computing Center supported by the Department of Energy, Office of Science, under Contract No. DE- AC0205CH11231. J.K.F. was also supported by the McDevitt bequest at Georgetown.

[1] A. Damascelli, Z. Hussain, and Z.-X. Shen, AngleResolved Photoemission Studies of the Cuprate Superconductors, Rev. Mod. Phys. 75, 473 (2003).

[2] T. Cuk, D. H. Lu, X. J. Zhou, Z.-X. Shen, T. P. Devereaux, and N. Nagaosa, A Review of Electron-Phonon Coupling Seen in the High- $T_{c}$ Superconductors by Angle-Resolved Photoemission Studies (ARPES), Phys. Status Solidi B 242, 11 (2005).

[3] P. V. Bogdanov, A. Lanzara, S. A. Kellar, X. J. Zhou, E. D. Lu, W. J. Zheng, G. Gu, J.-I. Shimoyama, K. Kishio, H. Ikeda et al., Evidence For An Energy Scale For Quasiparticle Dispersion in $\mathrm{Bi}_{2} \mathrm{Sr}_{2} \mathrm{CaCu}_{2} \mathrm{O}_{8}$, Phys. Rev. Lett. 85, 2581 (2000).

[4] P.D. Johnson, T. Valla, A. V. Fedorov, Z. Yusof, B. O. Wells, Q. Li, A.R. Moodenbaugh, G.D. Gu, N. Koshizuka, C. Kendziora et al., Doping and Temperature Dependence of the Mass Enhancement Observed in the Cuprate $\mathrm{Bi}_{2} \mathrm{Sr}_{2} \mathrm{CaCu}_{2} \mathrm{O}_{8+\delta}$, Phys. Rev. Lett. 87, 177007 (2001).

[5] A. Lanzara, P. V. Bogdanov, X. J. Zhou, S. A. Kellar, D. L. Feng, E. D. Lu, T. Yoshida, H. Eisaki, A. Fujimori, K. Kishio et al., Evidence for Ubiquitous Strong ElectronPhonon Coupling in High-Temperature Superconductors, Nature (London) 412, 510 (2001).

[6] A. Kaminski, M. Randeria, J.C. Campuzano, M.R. Norman, H. Fretwell, J. Mesot, T. Sato, T. Takahashi, and K. Kadowaki, Renormalization of Spectral Line Shape and Dispersion below $T_{c}$ in $\mathrm{Bi}_{2} \mathrm{Sr}_{2} \mathrm{CaCu}_{2} \mathrm{O}_{8+\delta}$, Phys. Rev. Lett. 86, 1070 (2001).
[7] D. S. Dessau, B. O. Wells, Z.-X. Shen, W. E. Spicer, A. J. Arko, R.S. List, D.B. Mitzi, and A. Kapitulnik, Anomalous Spectral Weight Transfer at the Superconducting Transition of $\mathrm{Bi}_{2} \mathrm{Sr}_{2} \mathrm{CaCu}_{2} \mathrm{O}_{8+\delta}$, Phys. Rev. Lett. 66, 2160 (1991).

[8] T. Cuk, F. Baumberger, D. H. Lu, N. Ingle, X. J. Zhou, H. Eisaki, N. Kaneko, Z. Hussain, T.P. Devereaux, N. Nagaosa et al., Coupling of the $B_{1 g}$ Phonon to the Antinodal Electronic States of $\mathrm{Bi}_{2} \mathrm{Sr}_{2} \mathrm{Ca}_{0.92} \mathrm{Y}_{0.08} \mathrm{Cu}_{2} \mathrm{O}_{8+\delta}$, Phys. Rev. Lett. 93, 117003 (2004).

[9] A.D. Gromko, A. V. Fedorov, Y.-D. Chuang, J. D. Koralek, Y. Aiura, Y. Yamaguchi, K. Oka, Y. Ando, and D.S. Dessau, Mass-Renormalized Electronic Excitations at $(\pi, 0)$ in the Superconducting State of $\mathrm{Bi}_{2} \mathrm{Sr}_{2} \mathrm{CaCu}_{2} \mathrm{O}_{8+\delta}$, Phys. Rev. B 68, 174520 (2003).

[10] T. K. Kim, A. A. Kordyuk, S. V. Borisenko, A. Koitzsch, M. Knupfer, H. Berger, and J. Fink, Doping Dependence of the Mass Enhancement in $(\mathrm{Pb}, \mathrm{Bi})_{2} \mathrm{Sr}_{2} \mathrm{CaCu}_{2} \mathrm{O}_{8}$ at the Antinodal Point in the Superconducting and Normal States, Phys. Rev. Lett. 91, 167002 (2003).

[11] T. Sato, H. Matsui, T. Takahashi, H. Ding, H.-B. Yang, S.-C. Wang, T. Fujii, T. Watanabe, A. Matsuda, T. Terashima et al., Observation of Band Renormalization

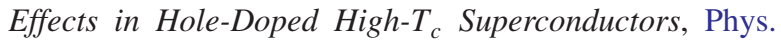
Rev. Lett. 91, 157003 (2003).

[12] M. R. Norman, H. Ding, J. C. Campuzano, T. Takeuchi, M. Randeria, T. Yokoya, T. Takahashi, T. Mochiku, and K. Kadowaki, Unusual Dispersion and Line Shape of the Superconducting State Spectra of $\mathrm{Bi}_{2} \mathrm{Sr}_{2} \mathrm{CaCu}_{2} \mathrm{O}_{8+\delta}$, Phys. Rev. Lett. 79, 3506 (1997).

[13] S. V. Borisenko, A. A. Kordyuk, V. Zabolotnyy, J. Geck, D. Inosov, A. Koitzsch, J. Fink, M. Knupfer, B. Büchner, V. Hinkov et al., Kinks, Nodal Bilayer Splitting, and Interband Scattering in $\mathrm{YBa}_{2} \mathrm{Cu}_{3} \mathrm{O}_{6+x}$, Phys. Rev. Lett. 96, 117004 (2006).

[14] S. V. Borisenko, A. A. Kordyuk, A. Koitzsch, J. Fink, J. Geck, V. Zabolotnyy, M. Knupfer, B. Büchner, H. Berger, M. Falub et al., Parity of the Pairing Bosons in a High-Temperature $\mathrm{Pb}-\mathrm{Bi}_{2} \mathrm{Sr}_{2} \mathrm{CaCu}_{2} \mathrm{O}_{8}$ Bilayer Superconductor by Angle-Resolved Photoemission Spectroscopy, Phys. Rev. Lett. 96, 067001 (2006).

[15] A. A. Kordyuk, S. V. Borisenko, V. B. Zabolotnyy, J. Geck, M. Knupfer, J. Fink, B. Büchner, C. T. Lin, B. Keimer, H. Berger et al., Constituents of the Quasiparticle Spectrum Along the Nodal Direction of High- $T_{c}$ Cuprates, Phys. Rev. Lett. 97, 017002 (2006).

[16] W. Meevasana, N. J. C. Ingle, D. H. Lu, J. R. Shi, F. Baumberger, K. M. Shen, W. S. Lee, T. Cuk, H. Eisaki, T.P. Devereaux et al., Doping Dependence of the Coupling of Electrons to Bosonic Modes in the SingleLayer High-Temperature $\mathrm{Bi}_{2} \mathrm{Sr}_{2} \mathrm{CuO}_{6}$ Superconductor, Phys. Rev. Lett. 96, 157003 (2006).

[17] X. J. Zhou, J. Shi, T. Yoshida, T. Cuk, W. L. Yang, V. Brouet, J. Nakamura, N. Mannella, S. Komiya, Y. Ando et al., Multiple Bosonic Mode Coupling in the Electron Self-Energy of $\left(\mathrm{La}_{2-x} \mathrm{Sr}_{x}\right) \mathrm{CuO}_{4}$, Phys. Rev. Lett. 95, 117001 (2005).

[18] J. Fink, A. Koitzsch, J. Geck, V. Zabolotnyy, M. Knupfer, B. Büchner, A. Chubukov, and H. Berger, Reevaluation of the Coupling to a Bosonic Mode of the Charge Carriers in 
(Bi, $\mathrm{Pb})_{2} \mathrm{Sr}_{2} \mathrm{CaCu}_{2} \mathrm{O}_{8+\delta}$ at the Antinodal Point, Phys. Rev. B 74, 165102 (2006).

[19] T. Dahm, V. Hinkov, S. V. Borisenko, A. A. Kordyuk, V. B. Zabolotnyy, J. Fink, B. Buchner, D. J. Scalapino, W. Hanke, and B. Keimer, Strength of the Spin-FluctuationMediated Pairing Interaction in a High-Temperature Superconductor, Nat. Phys. 5, 217 (2009).

[20] T. Valla, A. V. Fedorov, P. D. Johnson, B. O. Wells, S. L. Hulbert, Q. Li, G. D. Gu, and N. Koshizuka, Evidence for Quantum Critical Behavior in the Optimally Doped Cuprate $\mathrm{Bi}_{2} \mathrm{Sr}_{2} \mathrm{CaCu}_{2} \mathrm{O}_{8+\delta}$, Science 285, 2110 (1999).

[21] T. J. Reber, N.C. Plumb, Z. Sun, Y. Cao, Q. Wang, K. McElroy, H. Iwasawa, M. Arita, J. S. Wen, Z. J. Xu et al., The Origin and Non-Quasiparticle Nature of Fermi Arcs in $\mathrm{Bi}_{2} \mathrm{Sr}_{2} \mathrm{CaCu}_{2} \mathrm{O}_{8+\delta}$, Nat. Phys. 8, 606 (2012).

[22] P. B. Allen, Theory of Thermal Relaxation of Electrons in Metals, Phys. Rev. Lett. 59, 1460 (1987).

[23] S. D. Brorson, A. Kazeroonian, J.S. Moodera, D. W. Face, T. K. Cheng, E.P. Ippen, M.S. Dresselhaus, and G. Dresselhaus, Femtosecond Room-Temperature Measurement of the Electron-Phonon Coupling Constant $\gamma$ in Metallic Superconductors, Phys. Rev. Lett. 64, 2172 (1990).

[24] U. Bovensiepen, Coherent and Incoherent Excitations of the Gd(0001) Surface on Ultrafast Timescales, J. Phys. Condens. Matter 19, 083201 (2007).

[25] L. Perfetti, P. A. Loukakos, M. Lisowski, U. Bovensiepen, H. Eisaki, and M. Wolf, Ultrafast Electron Relaxation in Superconducting $\mathrm{Bi}_{2} \mathrm{Sr}_{2} \mathrm{CaCu}_{2} \mathrm{O}_{8+\delta}$ by Time-Resolved Photoelectron Spectroscopy, Phys. Rev. Lett. 99, 197001 (2007).

[26] R. Cortés, L. Rettig, Y. Yoshida, H. Eisaki, M. Wolf, and U. Bovensiepen, Momentum-Resolved Ultrafast Electron Dynamics in Superconducting $\mathrm{Bi}_{2} \mathrm{Sr}_{2} \mathrm{CaCu}_{2} \mathrm{O}_{8+\delta}$, Phys. Rev. Lett. 107, 097002 (2011).

[27] J. Graf, C. Jozwiak, C. L. Smallwood, H. Eisaki, R. A. Kaindl, D.-H. Lee, and A. Lanzara, Nodal Quasiparticle Meltdown in Ultrahigh-Resolution Pump-Probe AngleResolved Photoemission, Nat. Phys. 7, 805 (2011).

[28] L. Rettig, R. Cortés, S. Thirupathaiah, P. Gegenwart, H. S. Jeevan, M. Wolf, J. Fink, and U. Bovensiepen, Ultrafast Momentum-Dependent Response of Electrons in Antiferromagnetic $\mathrm{EuFe}_{2} \mathrm{As}_{2}$ Driven by Optical Excitation, Phys. Rev. Lett. 108, 097002 (2012).

[29] I. Avigo, R. Cortés, L. Rettig, S. Thirupathaiah, H. S. Jeevan, P. Gegenwart, T. Wolf, M. Ligges, M. Wolf, J. Fink et al., Coherent Excitations and Electron-Phonon Coupling in $\mathrm{Ba} / \mathrm{EuFe}_{2} \mathrm{As}_{2}$ Compounds Investigated by Femtosecond Time- and Angle-Resolved Photoemission Spectroscopy, J. Phys. Condens. Matter 25, 094003 (2013).

[30] A. F. Kemper, M. M. Korshunov, T.P. Devereaux, J. N. Fry, H.-P. Cheng, and P. J. Hirschfeld, Anisotropic Quasiparticle Lifetimes in Fe-Based Superconductors, Phys. Rev. B 83, 184516 (2011).

[31] T.P. Devereaux, T. Cuk, Z.-X. Shen, and N. Nagaosa, Anisotropic Electron-Phonon Interaction in the Cuprates, Phys. Rev. Lett. 93, 117004 (2004).

[32] D. Fausti, R. I. Tobey, N. Dean, S. Kaiser, A. Dienst, M. C. Hoffmann, S. Pyon, T. Takayama, H. Takagi, and A.
Cavalleri, Light-Induced Superconductivity in a StripeOrdered Cuprate, Science 331, 189 (2011).

[33] A. D. Caviglia, R. Scherwitzl, P. Popovich, W. Hu, H. Bromberger, R. Singla, M. Mitrano, M. C. Hoffmann, S. Kaiser, P. Zubko et al., Ultrafast Strain Engineering in Complex Oxide Heterostructures, Phys. Rev. Lett. 108, 136801 (2012).

[34] S. Wall, D. Wegkamp, L. Foglia, K. Appavoo, J. Nag, R. F. Haglund, J. Stähler, and M. Wolf, Ultrafast Changes in Lattice Symmetry Probed by Coherent Phonons, Nat. Commun. 3, 721 (2012).

[35] N. Tsuji, T. Oka, P. Werner, and H. Aoki, Dynamical Band Flipping in Fermionic Lattice Systems: An ac-FieldDriven Change of the Interaction from Repulsive to Attractive, Phys. Rev. Lett. 106, 236401 (2011).

[36] S. Hellmann, T. Rohwer, M. Kalläne, K. Hanff, C. Sohrt, A. Stange, A. Carr, M. M. Murnane, H. C. Kapteyn, L. Kipp et al., Time-Domain Classification of ChargeDensity-Wave Insulators, Nat. Commun. 3, 1069 (2012).

[37] W.S. Fann, R. Storz, H. W. K. Tom, and J. Bokor, Direct Measurement of Nonequilibrium Electron-Energy Distributions in Subpicosecond Laser-Heated Gold Films, Phys. Rev. Lett. 68, 2834 (1992).

[38] M. Hentschel, R. Kienberger, C. Spielmann, G. A. Reider, N. Milosevic, T. Brabec, P. M. Hentschel, R. Kienberger, C. Spielmann, G. A. Reider et al., Attosecond Metrology, Nature (London) 414, 509 (2001).

[39] F. Schmitt, P. S. Kirchmann, U. Bovensiepen, R. G. Moore, L. Rettig, M. Krenz, J.-H. Chu, N. Ru, L. Perfetti, D. H. Lu et al., Transient Electronic Structure and Melting of a Charge Density Wave in $\mathrm{TbTe}_{3}$, Science 321, 1649 (2008).

[40] L. Perfetti, P. A. Loukakos, M. Lisowski, U. Bovensiepen, H. Berger, S. Biermann, P. S. Cornaglia, A. Georges, and M. Wolf, Time Evolution of the Electronic Structure of $1 \mathrm{~T}-\mathrm{TaS}_{2}$ through the Insulator-Metal Transition, Phys. Rev. Lett. 97, 067402 (2006).

[41] L. Perfetti, P. A. Loukakos, M. Lisowski, U. Bovensiepen, M. Wolf, H. Berger, S. Biermann, and A. Georges, Femtosecond Dynamics of Electronic States in the Mott Insulator $1 \mathrm{~T}-\mathrm{TaS}_{2}$ by Time Resolved Photoelectron Spectroscopy, New J. Phys. 10, 053019 (2008).

[42] G. L. Dakovski, Y. Li, T. Durakiewicz, and G. Rodriguez, Tunable Ultrafast Extreme Ultraviolet Source for Timeand Angle-Resolved Photoemission Spectroscopy, Rev. Sci. Instrum. 81, 073108 (2010).

[43] J. A. Sobota, S. Yang, J. G. Analytis, Y. L. Chen, I. R. Fisher, P.S. Kirchmann, and Z.-X. Shen, Ultrafast Optical Excitation of a Persistent Surface-State Population in the Topological Insulator $\mathrm{Bi}_{2} \mathrm{Se}_{3}$, Phys. Rev. Lett. 108, 117403 (2012).

[44] J. W. McIver, D. Hsieh, H. Steinberg, P. Jarillo-Herrero, and N. Gedik, Control Over Topological Insulator Photocurrents with Light Polarization, Nat. Nanotechnol. 7, 96 (2011).

[45] C. M. Heyl, J. Güdde, A. L'Huillier, and U. Höfer, HighOrder Harmonic Generation with $\mu \mathrm{J}$ Laser Pulses at High Repetition Rates, J. Phys. B 45, 074020 (2012).

[46] S. Hellmann, C. Sohrt, M. Beye, T. Rohwer, F. Sorgenfrei, M. Marczynski-Bühlow, M. Kalläne, H. Redlin, F. Hennies, M. Bauer et al., Time-Resolved X-Ray Photoelectron 
Spectroscopy at FLASH, New J. Phys. 14, 013062 (2012).

[47] G. Rohde, T. Rohwer, C. Sohrt, A. Stange, S. Hellmann, L. Yang, K. Hanff, A. Carr, M. Murnane, H. Kapteyn et al., Tracking the Relaxation Pathway of Photo-Excited Electrons in $1 \mathrm{~T}-\mathrm{TiSe}_{2}$, Eur. Phys. J. Spec. Top. 222, 997 (2013).

[48] See Supplemental Material at http://link.aps.org/ supplemental/10.1103/PhysRevX.3.041033 for a discussion of the influence of the probe pulse width, the breakdown of an effective temperature description, details of the relaxation rates, and the inclusion of electronelectron scattering.

[49] R. Peierls, Zur Theorie des Diamagnetismus von Leitungselektronen, Z. Phys. 80, 763 (1933).

[50] A.P. Jauho and J. W. Wilkins, Theory of High-ElectricField Quantum Transport for Electron-Resonant Impurity Systems, Phys. Rev. B 29, 1919 (1984).

[51] A.B. Migdal, Zh. Eksp. Teor. Fiz. 34, 1438 (1958) [Interaction Between Electrons and Lattice Vibrations in a Normal Metal, Sov. Phys. JETP 7, 996 (1958)].

[52] M. Eckstein, M. Kollar, and P. Werner, Interaction Quench in the Hubbard Model: Relaxation of the Spectral Function and the Optical Conductivity, Phys. Rev. B 81, 115131 (2010).

[53] J. K. Freericks, H.R. Krishnamurthy, and T. Pruschke, Theoretical Description of Time-Resolved Photoemission Spectroscopy: Application to Pump-Probe Experiments, Phys. Rev. Lett. 102, 136401 (2009).

[54] V. Turkowski and J. K. Freericks, Nonequilibrium Dynamical Mean-Field Theory of Strongly Correlated Electrons, in: Strongly Correlated Systems: Coherence and Entanglement (World Scientific, Singapore, 2007), pp. 187-210.

[55] T. Holstein, Studies of Polaron Motion: Part I. The Molecular-Crystal Model, Ann. Phys. (N.Y.) 8, 325 (1959).

[56] G. D. Mahan, Many Particle Physics (Springer, New York, 2010).

[57] J. K. Freericks, V.M. Turkowski, and V. Zlatic, Nonequilibrium Dynamical Mean-Field Theory, Phys. Rev. Lett. 97, 26408 (2006).

[58] J.K. Freericks, Quenching Bloch Oscillations in a Strongly Correlated Material: Nonequilibrium Dynamical Mean-Field Theory, Phys. Rev. B 77, 075109 (2008).

[59] M. Wagner, Expansions of Nonequilibrium Green's Functions, Phys. Rev. B 44, 6104 (1991).

[60] N.W. Ashcroft and N.D. Mermin, Solid State Physics (Brooks Cole, Pacific Grove, CA, 1976).

[61] S. Winnerl, M. Orlita, P. Plochocka, P. Kossacki, M. Potemski, T. Winzer, E. Malic, A. Knorr, M. Sprinkle, C. Berger et al., Carrier Relaxation in Epitaxial Graphene
Photoexcited Near the Dirac Point, Phys. Rev. Lett. 107, 237401 (2011).

[62] S. Gilbertson, T. Durakiewicz, J.-X. Zhu, A. D. Mohite, A. Dattelbaum, and G. Rodriguez, Direct Measurement of Quasiparticle Lifetimes in Graphene Using Time-Resolved Photoemission, J. Vac. Sci. Technol. B 30, 03 D116 (2012).

[63] S. Winnerl, F. Göttfert, M. Mittendorff, H. Schneider, M. Helm, T. Winzer, E. Malic, A. Knorr, M. Orlita, M. Potemski et al., Time-Resolved Spectroscopy on Epitaxial Graphene in the Infrared Spectral Range: Relaxation Dynamics and Saturation Behavior, J. Phys. Condens. Matter 25, 054202 (2013).

[64] J. C. Johannsen, S. Ulstrup, F. Cilento, A. Crepaldi, M. Zacchigna, C. Cacho, I. C.E. Turcu, E. Springate, F. Fromm, C. Raidel et al., Direct View of Hot Carrier Dynamics in Graphene, Phys. Rev. Lett. 111, 027403 (2013).

[65] I. Gierz, J. C. Petersen, M. Mitrano, C. Cacho, E. Turcu, E. Springate, A. Stöhr, A. Köhler, U. Starke, and A. Cavalleri, Snapshots of Non-Equilibrium Dirac Carrier Distributions in Graphene, Nat. Mater. 12, 1119 (2013).

[66] C. L. Smallwood, J.P. Hinton, C. Jozwiak, W. Zhang, J. D. Koralek, H. Eisaki, D.-H. Lee, J. Orenstein, and A. Lanzara, Tracking Cooper Pairs in a Cuprate Superconductor by Ultrafast Angle-Resolved Photoemission, Science 336, 1137 (2012).

[67] S. I. Anisimov, B. L. Kapeliovich, and T. L. Perelman, Electron Emission from Metal Surfaces Exposed to Ultrashort Laser Pulses, Sov. Phys. JETP 39, 375 (1974).

[68] E. E. Mendez and G. Bastard, Wannier-Stark Ladders and Bloch Oscillations in Superlattices, Phys. Today 46, 34 (1993).

[69] B. Moritz, T.P. Devereaux, and J. K. Freericks, TimeResolved Photoemission of Correlated Electrons Driven out of Equilibrium, Phys. Rev. B 81, 165112 (2010).

[70] G. D. Mahan, Quantum Transport Equation for Electric and Magnetic Fields, Phys. Rep. 145, 251 (1987).

[71] A. F. Kemper, M. Sentef, B. Moritz, C. C. Kao, Z. X. Shen, J.K. Freericks, and T.P. Devereaux, Mapping of Unoccupied States and Relevant Bosonic Modes via the Time-Dependent Momentum Distribution, Phys. Rev. B 87, 235139 (2013).

[72] J. K. Freericks and V.M. Turkowski, Steady-State Nonequilibrium Dynamical Mean-Field Theory and the Quantum Boltzmann Equation, J. Phys. Conf. Ser. 35, 39 (2006).

[73] B. Moritz, A. F. Kemper, M. Sentef, T. P. Devereaux, and J. K. Freericks, Electron-Mediated Relaxation Following Ultrafast Pumping of Strongly Correlated Materials: Model Evidence of a Correlation-Tuned Crossover between Thermal and Nonthermal States, Phys. Rev. Lett. 111, 077401 (2013). 